\title{
COUTAPORTLA, GÉNERO ENDÉMICO DE MÉXICO, LORENCEA (RUBIACEAE) ENDÉMICO EN MESOAMÉRICA
}

\author{
A. L. BorHIDI \\ Instituto de Biología, Universidad de Pécs, H-7624 Pécs, Ifjúság útja 6, Hungría \\ E-mail: borhidi@gamma.ttk.pte.hu
}

(Received 27 November, 2017; Accepted 5 January, 2018)

A new genus was described based on Portlandia guatemalensis Standl. of uncertain taxonomic position. The proposal of D. H. Lorence to place it into the genus Coutaportla Urb. as C. guatemalensis (Standl.) Lorence has not been supported by the discovery of a new species of Coutaportla (C. pailensis) nor by the molecular taxonomic studies of the group either. The differences of floral morphological, ovary and seed anatomical characters existing between the actually known three Coutaportla species of Lorence are the following: 1.) Placentation: all the three species belong to the Portlandia-complex (Urban 1923, Aiello 1979). Within the complex the placentation is one of the more important, fundamental feature in distinction and separation of the genera (Urban, 1923, Aiello 1979). Coutaportla has a unique form of placentation, the Coutaportla-type with a central-horizontal quadrangular placenta with determined number and position of ovules: 2-3 ascendent and 2-3 colgate separately positioned ones (Aiello 1979). This type of placentation is found only in Coutaportla ghisbreghtiana (the type species of the genus) and in C. pailensis. Coutaportla guatemalensis (Standl.) Lorence has a different form of placentation, with a basal-ascendent placenta and vertically arranged undetermined number of tile-likely arranged ovules, which is of Hintonia-type (Lorence 1986, Borhidi 2003). 2.) Morphological differences: five-six further important differences are found in the stipular, floral and seed morphology of the two Mexican (C. ghisbreghtiana and C. pailensis) and the one Mesoamerican (C. guatemalensis) species (Borhidi 2003, see below in the table). 3.) Molecular differences: the two species group have distant placements in the molecular cladograms of Chiococceae tribe belonging to two different major clades (Motley et al. 2005, Manns and Bremer 2010). All these differences seemed to give satisfactory amount of arguments for the separation of C. guatemalensis as an independent monotypic genus, the Lorencea Borhidi 2003, as endemic to the Mesoamerican flora, dedicated to the honor of D. H. Lorence, who has made numerous important discoveries and successful efforts to promote our knowledge about the Mexican and Central American Rubiaceae. The presented arguments convinced the experts of the World checklist of Rubiaceae to accept it as a valid new genus (Govaerts et al. 2006). As an obvious phytogeographical consequence of all these previous investigations, Coutaportla Urb. turned to be a Mexican endemic genus with two species living in the states Coahuila, Puebla and Oaxaca, of México and Lorencea Borhidi a monotypic endemic one of Mesoamerica, living in the states Chiapas of México and Guatemala. It was a surprise, that in the Flora Mesoamericana (2012): appeared the genus Coutaportla represented by C. guatemalensis (Standl.) Lorence, reducing Lorencea into synonymy with the following sentence: "No hay características de $C$. guatemalensis que apoyan su separación como género monotípico, como propuso Borhidi", which is obviously an orbital lie with full knowledge of the above detailed facts. The treatment of the genus Coutaportla en la Flora Mesoamérica is a collection of inadmissible taxonomic inconsequences, starting with the modified generic protologue of 
Coutaportla by Ochoterena 2012 non Urban 1923, but attributed to Urban without amendment, which is a falsification of the original description of Urban based on a new type and including alien elements without new original studies (see Flora Mesoamericana 4.2: 69). Not to mention the absence of the obligatory citation of the criticised paper of Borhidi, and the fundamental publications of Aiello (1979), Motley et al. (2005), Borhidi (2006), and Manns and Bremer (2010). Therefore, the critical treatment of the genus Coutaportla with the re-establishment of Lorencea is a scientifically well-based, obvious, taxonomic necessity.

Key words: Coutaportla, endemism, Lorencea, molecular evidences, morphology, re-establishment

\section{INTRODUCCIÓN}

La delimitación de los géneros en la familia Rubiaceae es a menudo una tarea muy dificil, porque mucho de ellos tiene semejanza superficial muy impresionante, mientras sus relaciones genéticas están basadas en caracteres anatómicos escondidos. Para revelar y reconocer estos caracteres decisivos necesitamos utilizar muchas veces técnicas electron-microscópicas,bioquímicas etc. En los ultimos años dos complejos de géneros sirvieron cómo ejemplares instructivos, "el complejo de Portlandia" (Aiello 1979) y el complejo de Rondeletia (Delprete 1999). El complejo de Portlandia a la que pertenecen actualmente géneros distribuidos en dos tribús (Cinchoneae y Condamineae) fue estudiado por primera vez según criterios modernos por el genial Ignatius Urban en 1923. El utilizó la dehiscencia del fruto y la placentación como criterios fundamentales y separó cuatro géneros: Schmidtottia Urb., Siemensia Urb., Nernstia Urb. y Coutaportla Urb. (Este último nombre genérico compusó de los nombres de géneros Coutarea y Portlandia). Su idea fue seguida utilizando técnicas más finas en el trabajo excelente de Aiello, quién confirmó la existencia de los géneros de Urban y añadió otro cuatro géneros nuevos más: Ceuthocarpus Aiello, Cubanola Aiello, Thogsennia Aiello, y Osa Aiello. Una especie dudosa se quedó fuera de la revisión de Aiello por no haber tenido frutos maduros, y esta es la Portlandia guatemalensis Standl. Standley expresó sus dudas en cuanto a la posición correcta de la especie reiteradamente, en la descripción (1928) y en la Flora de Guatemala (Standley y Williams 1975). Aiello llegó a la conclusión, que la especie seguramente no es una Portlandia, y teniendo fruto muy jóven, suppusó su pertenencia posible a Coutarea o a Coutaportla, o cómo una representante de un género no descrito. Es interesante notar, que un proceso parecido occurrió relacionado al complejo de Rondeletia, donde el estudio de Steyermark (1967) inició la división del complejo por el reconocimiento de los géneros Arachnothryx y Rogiera, seguido por la descripción de cuatro géneros nuevos por el autor de este artículo y sus colaboradores (Acunaeanthus 1980, Roigella 1981, Suberanthus1981 y Javorkaea 1983). La historia de la Portlandia de relación taxonómica incierta continua con el articulo de Lorence (1986) sobre 
la identidad de la Portlandia guatemalensis. El tenía posibilidad de estudiar material abundante fructificado de la colecta de Breedlove, que permitió realizar estudios comparativos entre los géneros Coutarea, Coutaportla y Portlandia y concluyó, que la Portlandia guatemalensis esta más cercana al género Coutaportla por tener semillas discoideas, exaladas con testa reticulada pocas por lóculos. A base de estos criterios Lorence incluyó la especie en cuestión al género Coutaportla creando la combinación: Coutaportla guatemalensis (Standl.) Lorence.

\section{DISCUSIÓN}

Lamentablemente la combinación nueva creada por Lorence no encontró la posición correcta de la especie en cuestión, porqué dejó fuera de la atención una serie de diferencias morfológicas, anatómicas y moleculares existentes entre Coutaportla ghiesbreghtiana y C. guatemalensis. Estos son los siguientes.

1. Morfología de la placenta. Cómo Aiello enfatizó, la forma de la placenta en el grupo Portlandia es un caracter fundamental. Cada género estudiado por Aiello tiene otra forma de placenta. La Coutaportla ghiesbreghtiana tiene una placenta laminar de forma cuadrangular saliendo horizontalmente del centro del septo del ovario, que divide el lóculo en dos mitades, una parte superior y una inferior (Fig. 1). La Coutaportla guatemalensis tiene una placenta linear de dirección vertical saliendo del ángulo basal del lóculo (Fig. 2). Las dos placentas son completamente diferentes. La placentación basal es un fenómeno muy raro, en la tribú Chiococceae. En Rondeletieae la placenta basal es un caracter único del género Suberanthus.

2. Morfología de la placentación. En Coutaportla ghiesbreghtiana la placenta tiene una inserción central en el septo del ovario y extiende en dirección horizontal. Los óvulos y las semillas están sentadas en dos series. En la parte superior los óvulos están ascendentes, en la parte inferior están colgantes, y en ambos mitades del lóculo están situadas separadamente en número determinado 2 o 3 en ambos mitades (Fig. 1). En la Coutaportla guatemalensis los óvulos están sentados en una serie y todos son ascendentes, verticales. No están situados separados, sino imbricados y en número indeterminado (Fig. 2). Las dos placentaciones son completamente distintas.

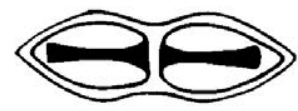

a
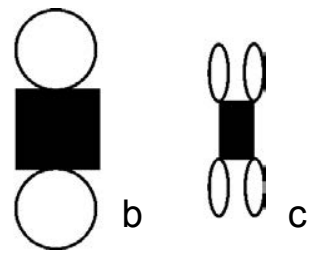

Fig. 1. Placenta y placentación en Coutaportla ghiesbreghtiana según A. Aiello $(\mathrm{a}=$ forma de la placenta en sección transversal; $b=$ posición de las semillas en sección perpendicular al septo, son ascendentes y colgantes; c = posición de las semillas en sección paralela al septo) 
Estudiabamos la placenta y la placentación en el ovario y frutos de Coutaportla pailensis, y encontramos, que en ambos criterios son idénticos con $C$. ghiesbreghtiana y distintos de C. guatemalensis. La monografista de la Flora Mesoamericana no estudió esta tercera especie.

3. Testa de las semillas. En la decisión de Lorence la forma parecida de las semillas tiene un papel importante, aunque en el tamaño de las semillas hay una diferencia notable. Peró sabemos también, que en las tribús Chiococceae y Rondeletieae la matiz celular de la testa es un caracter muy importante también, que puede caracterizar géneros con varias decenas de especies (Borhidi et al. 2004). En Coutaportla ghisbreghtiana las células de la testa tienen la pared horizontal concava y lisa, la pared perpendicular con un tuberculo terminal en cada célula. En Coutaportla guatemalensis las células de la testa tienen el pared horizontal densamente tuberculado y el pared perpendicular liso. Los dos matizes son completamente distintos (Fig. 3).

4. Otras diferencias. En la tabla 2 del articulo de Lorence enumera otros 12 caracteres, en que la dos especies difieren. Coutaportla ghisbreghtiana tiene estipular interpeciolares, libres, corola 4-mera - criterio acentuado en protólogo de Urban! - filamentos hirsutos en todo su largo, lóbulos del cáliz planos, glandulosos en el margen, glabros por dentro, mientras Coutaportla guatemalensis tiene estipulas intrapeciolares, connadas en la base, corola 5-mera, filamentos pelosos sólo en la base, lóbulos del cáliz canaliculado, eglanduloso, peloso por dentro.
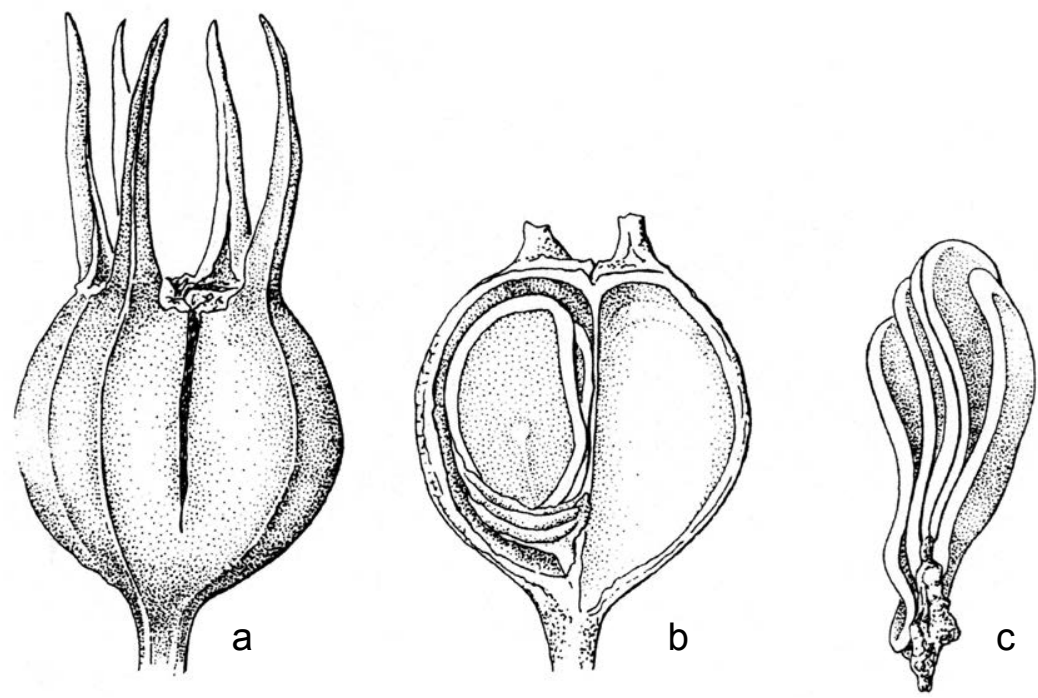

Fig. 2. Cápsula y semillas en Coutaportla guatemalensis según D. H. Lorence ( $a=$ cápsula, b = vista lateral con la pared perpendicular removido, mostrando el septo y la placenta con semillas de un lóculo (con algunas removidas); c = semillas en posición vertical, imbricadas en la placenta) 
Tabla 1

Caracteres diferenciales entre los géneros Coutaportla y Lorencea

\begin{tabular}{|c|c|c|}
\hline Caracter & $\begin{array}{l}\text { Coutaportla ghisbreghtiana y } \\
\text { C. pailensis }\end{array}$ & Lorencea guatemalensis \\
\hline Talla & arbusto de $1-3 \mathrm{~m}$ & arbol mediano de 9-19 m \\
\hline Estípula & interpeciolar, libre en la base & $\begin{array}{l}\text { intrapeciolar, connada en la } \\
\text { base }\end{array}$ \\
\hline \multicolumn{3}{|l|}{ Hojas } \\
\hline - tamaño & $0.4-5 \times 0.2-1.8 \mathrm{~cm}$ & $11-22 \times 3-9 \mathrm{~cm}$ \\
\hline - nervios laterales & $\begin{array}{l}2-5 \text { pares obsoletos o incon- } \\
\text { spicuos }\end{array}$ & $\begin{array}{l}\text { 7-10 pares impresos en el haz, } \\
\text { prominentes en el envés }\end{array}$ \\
\hline - axilas de nervios & planos y glabros & domaciados y barbudos \\
\hline - textura & coriácea & cartácea \\
\hline - color & concolor & discolor \\
\hline Inflorescencia & terminal & axilar (y terminal) \\
\hline Flores & 4-meras & 5-meras (rara vez 4-meras) \\
\hline Lóbulos del cáliz & plano & carinato en el dorso \\
\hline - cara interior & glabro & canaliculato e hirsutico \\
\hline - margen & $\begin{array}{l}\text { denticulado-glanduloso en } \\
\text { la base }\end{array}$ & entero, sin glándulas \\
\hline Corola, tamaño & $1-2.5 \mathrm{~cm}$ & $2.5-3.5 \mathrm{~cm}$ \\
\hline - vernación de lóbulos & estrechamente imbricado & induplicado imbricado \\
\hline Placenta & cuadrangular & linear \\
\hline - posición & central, horizontal & basal, vertical \\
\hline Óvulos & 4-6, separados & pocos, imbricados \\
\hline posición & ascendentes y descendentes & todos ascendentes \\
\hline Cápsula, tamaño & $4-10 \times 3-7 \mathrm{~mm}$ & $10-14 \times 12-16 \mathrm{~mm}$ \\
\hline Semillas, tamaño & $2-3.5 \mathrm{~mm}$ & $6-8 \mathrm{~mm}$ \\
\hline \multicolumn{3}{|l|}{ Células de la testa } \\
\hline - pared horizontal & concava, lisa plana & densamente tuberculada \\
\hline - pared perpendicular & con un tuberculo grande & lisa, sin ornamentación \\
\hline
\end{tabular}

En cuanto a los criterios morfológicos aqui enumerados hicimos estudios comparativos sobre ejemplares de C. pailensis Villareal también y encontramos, que en estas características C. ghiesbreghtiana y C. pailensis son semejantes, mientras los ejemplares de $C$. guatemalensis son diferentes. Las diferencias morfológicas están presentadas en la Tabla 1.

5. La consistencia del género Coutaportla. En 1987 se describió una nueva especie bajo el nombre Coutaportla pailensis Villarreal. Cuando en un género 
de dos especies aparece una tercera, esta nueva cambia la relación de las dos anteriores. Si es una especie intermediaria entre las dos anteriormente conocidas, la consistencia del género se aumenta con la nueva especie. Si la nueva especie tiene caracteres extremos en la cercania de una, con este se aumenta la diferencia entre las dos antes conocidas y destabiliza el género. Esta ultima alternativa es el caso con el descubrimiento de la Coutaportla pailensis, que es una planta más micrófila, más micrantha, más xeromorfa que los dos demás, peró tiene todos los caracteres listados más arriba, en que Coutaportla ghiesbreghtiana difiere de la Coutaportla guatemalensis. Pués, el descubrimiento de la Coutaportla pailensis Villareal aumentó la estabilidad del género Coutaportla Urb. (1923) en sentido original y destabilizó la Coutaportla en sentido de Lorence (1986) y de Ochoterena (2012) y justificó la decisión de Borhidi peresentado aqui reiteradamente sobre la existencia del género autónomo Lorencea.

6. El análisis molecular. En los trabajos de Rova (1999) figuras 3, y en Rova et al. (1999) fig. 7 se nota claramente, que Coutaportla ghisbreghtiana y Coutaportla guatemalensis se encuentran en el termino de dos ramas individuales largas, que significa una distancia muy larga, más larga que la mayoria de los géneros existentes en los cladogramos. La distancia genética molecular ha sido confirmado por los estudios de Motley et al. (2005), y esta decisión ha sido apoyado más tarde en la revisión de los estudios moleculares de la subfamilia Cinchonoideae por Manns y Bremer (2010). Ellas presentan claramente en el cladograma de los géneros del tribú Chiococceae que Coutaportla ghisbreghtiana y Lorencea guatemalensis están posicionadas en distancia notable de dos grupos de clados distintos (Figs 4-5).

Sumando todos estos argumentos arriba discutidos consideramos, que hay diferencias suficientes para re-establecer el género Lorencea a base de la Lo-
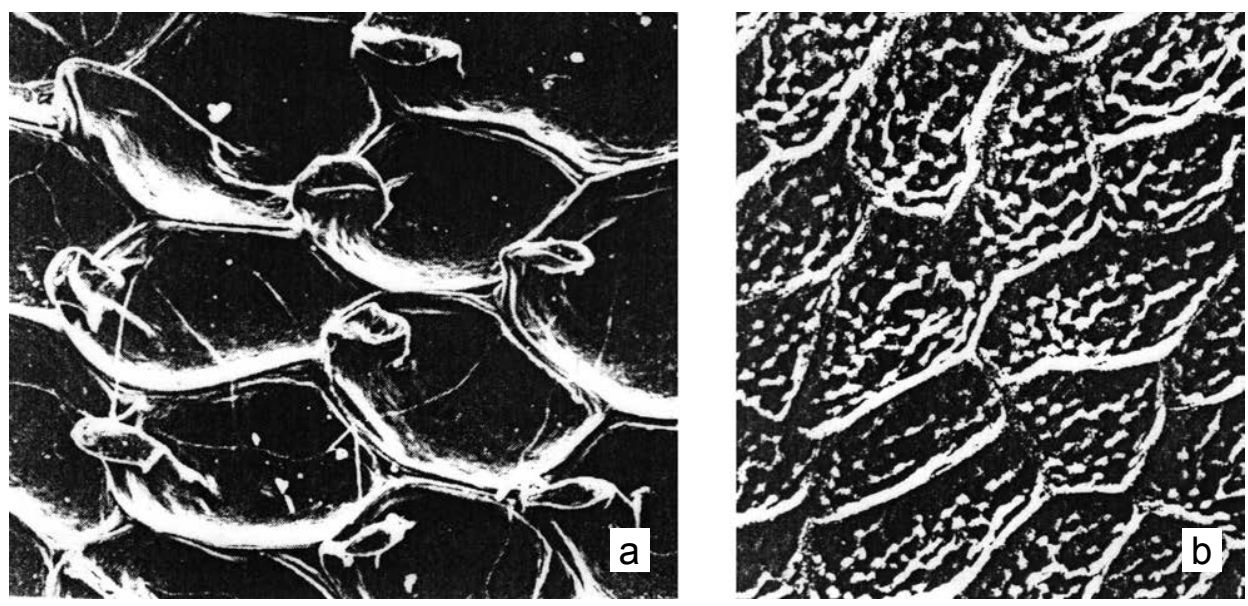

Fig. 3. Matiz celular de las semillas de las especies estudiadas; a = Coutaportla ghiesbreghtiana, según Aiello 1979, b = Lorencea guatemalensis, según Lorence 1986 
rencea guatemalensis (Standl.) Borhidi, con la caracterización publicada y detallada en 2003 y 2006, y como fue aceptado y legalizado por el World Checklist of Rubiaceae (Govaerts et al. 2006) en la edición de 2006 aceptó el género Lorencea, cómo género válido, endémico monotípico de la flora de Mesoamérica.

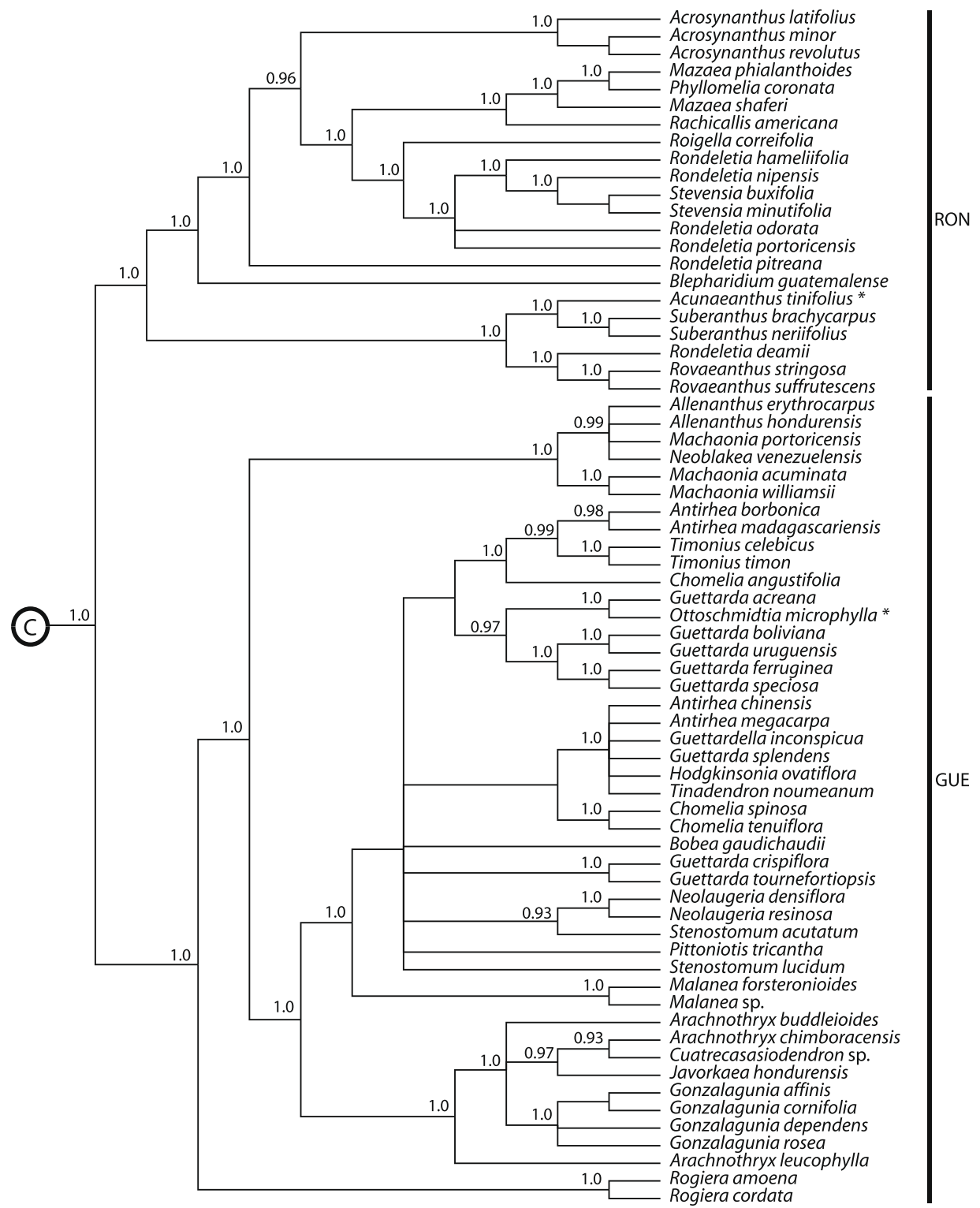

Fig. 4. Posición de Coutaportla ghiesbreghtiana y Lorencea guatemalensis en el cladograma molecular de las tribus Condamineae y Chiococceae, según Manns y B. Bremer (2010) 
Después de todo esto apareció el género Coutaportla en la Flora Mesoamericana representado por Coutaportla guatemalensis (Standl.) Lorence, con una caracterización grandemente modificando el protólogo original de Urban, produciendo un mixtura ilegítima de las caracresticas de los dos géneros distintos, sin estudio original y sin emendación necesaria, con el argumento siguiente: "No hay caracteristicas de C. guatemalensis, que apoyan su separación como género monotípico, como propuso Borhidi" que obviamente no es verdad, pero suguiere, que todo esto que Ustedes han leído arriba sobre las diferencias morfológicas, anatómicas y moleculares existentes entre los taxones cuestionados, taxonómicamente es nada - según la especialista invitada de la Flora Mesoamericana. Y para la mayor gloria de la objectividad científica, - influido con esta "argumentación concienzuda y detallada" - el World checklist of Rubiaceae retiró la validez del género Lorencea en 2012.

Estoy convencido en poder de los argumentos detallados arriba, que tengo que insistir en el asunto de la separación de los géneros Coutaportla y Lorencea, como están caracterizados en los dos ediciones de las Rubiáceas de México (Borhidi 2006, 2012).

\section{Coutaportla Urban 1923}

Coutaportla Urb., Symb. Antill. 9: 146 (1923). Tipo: Coutaportla ghiesbreghtiana (Baill.) Urb.

Arbustos o arbolitos hermafroditas, glabros o pubescentes, a menudo resinosos. Estípulas interpeciolares anchamente triangulares, agudas a cuspidadas, resinosas, persistentes. Hojas coriáceas, lustrosas. Flores grandes,

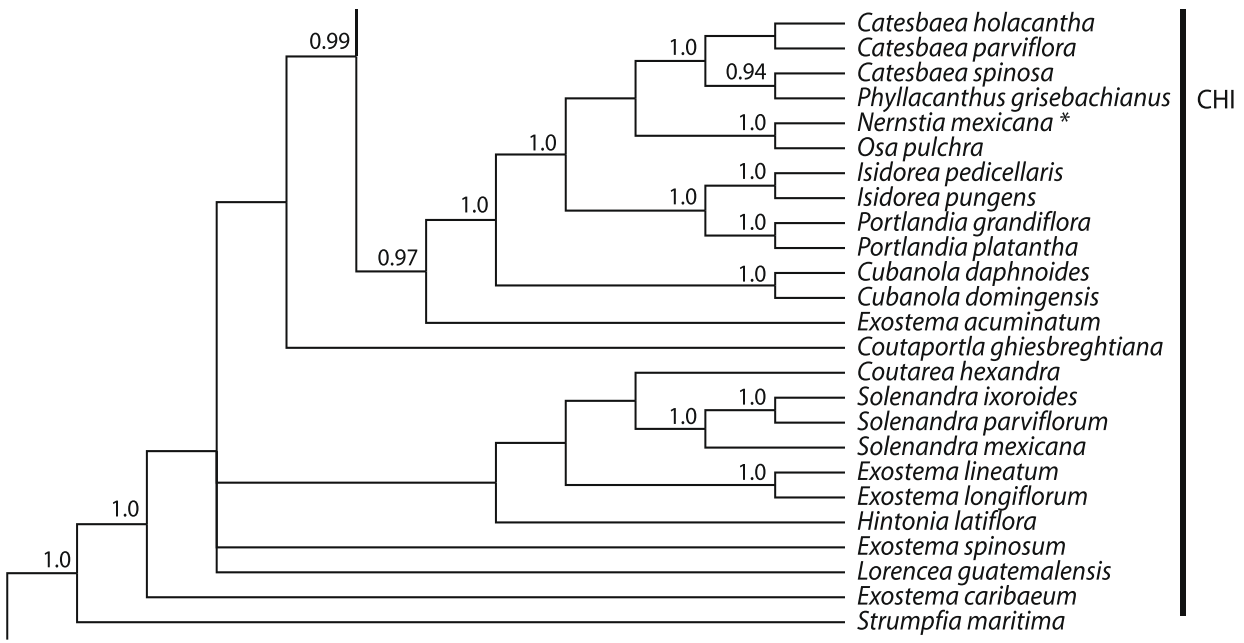

Fig. 5. Fragmento aumentado del cladograma anterior 
vistosas, axilares, numerosas o agrupadas en grupos laterales bracteosas. Hipanto turbinado, cáliz 4-lobulado, lóbulos alargados con glándulas rojas en el margen de la base, persistentes. Corola embudada, blanca, tubo 4-ánguloso, 2 a 3-veces más largo que los lóbulos, $2.5 \mathrm{~cm}$ de ancho arriba, lóbulos 4 , ovados, deltados o anchamente triangulares, estrechamente imbricados. Estambres 4, filamentos vellosos en toda su longitud, libres en la base. Anteras largas, lineares, basifijas. Fruto en cápsula oval-orbicular, fuertemente comprimida, 2 -surcada, obscuramente costada, redondeada en ambos extremos. Placenta central, horizontal dividiendo la celda en dos mitades, semillas por parte ascendentes y colgantes, 4 a 6 por lóculo. Semillas discoideas, comprimidas, engrosadas en el margen.

Género endémico de México con 2 especies.

- Hojas de 2 a $5 \mathrm{~cm}$ de largo, oblongo-elípticas u oblongo-lanceoladas, agudas y apiculadas en el ápice C. ghiesbreghtiana

- Hojas de 0.4 a 1.5 cm de largo, elíptico-orbiculares, coriáceas, enervias, redondeadas y mucronadas en el ápice $\quad$ C. pailensis

1. Coutaportla ghiesbreghtiana (Baill.) Urb., Symb. Ant. 9: 147 (1923). Bas.: Portlandia ghiesbreghtiana Baill., Adansonia 12: 300 (1879). - Tipo: México: Oaxaca, près de Hacienda de Huijastla, au bords des ravins, 1842-43, Ghiesbreght 27; holotipo: P!, foto: MEXU!; isotipo: US n.v., foto: MEXU! - Distr.: México: Oaxaca, Puebla, endémica.

Arbusto glabro con ramas verdes o negruzcas, gruesas o delgadas, erectas o colgantes, ramitas verdes y algo resinosas, entrenudos cortos. Estípulas anchamente triangulares, $2 \mathrm{~mm}$ de largo, agudas o cuspidadas, verdes y resinosas; pecíolos gruesos, 2 a $3 \mathrm{~mm}$ de largo. Hojas elípticas u oval-elípticas, de 1.7 a 4.5 por 0.8 a $2 \mathrm{~cm}$, aguda a acutiúscula en el ápice, cuneada en la base, coriácea, lustrosa en el haz, ligeramente más pálida y mate en el envés, el margen engrosado y plano, la costa subprominente, nervios laterales obsoletos. Flores axilares, numerosas, pedicelos delgados, 4 a $8 \mathrm{~mm}$ de largo. Hipanto de $3 \mathrm{~mm}$ de largo; lóbulos del cáliz 4, linear-subulados, 4 a $5 \mathrm{~mm}$ de largo, atenuados y agudos, glanduloso-denticulados en la base. Corola anchamente embudada, 2.2 a $2.5 \mathrm{~cm}$ de largo, blanco, tubo 6 a $7 \mathrm{~mm}$ de largo, 4-ánguloso, el limbo 2 a $2.5 \mathrm{~cm}$ de ancho, lóbulos 4, anchamente aovado-deltadas, 8 a 10 $\mathrm{mm}$ de largo, obtusos o acutiúsculos. Estambres 4, filamentos vellosos, anteras lineares, ca. $8 \mathrm{~mm}$ de largo. Cápsula oval-orbicular, 7 a $11 \mathrm{~mm}$ de largo y de ancho en el ápice, fuertemente comprimida, 2-sulcada, obscuramente costada, redondeada en ambos extremos. Semillas discoidales, comprimidas, engrosadas en el margen. 
2. Coutaportla pailensis Villareal, Sida 12: 223, fig. 1 (1987). - (Fig. 6) - Tipo: México: Coahuila, Mun. Ramos Arizpe, Sierra de la Paila, Cañón de Loma Prieta, al NW de Hipólito, 1800 m, J. A. Villáreal et al. 3305; holotipo: MEXU!, isotipos: ANSM n.v., ENCB!, TEX n.v. - Distr.: México: Coahuila, endémica.

Arbusto redondeado, muy ramoso, 10 a $40 \mathrm{~cm}$ de alto; tallos cilíndricos, menudamente pubescentes, nudos ensanchados, entrenudos 1 a $8 \mathrm{~mm}$ de largo. Estípulas interpeciolares, anchamente deltadas, persistentes, 0.6 a $0.8 \mathrm{~mm}$ de largo, 1.2 a $1.5 \mathrm{~mm}$ de ancho, coléteres marginales 1 a 4 pares y un apical. Hojas opuestas; pecíolos cortos, $0.5 \mathrm{a} 2 \mathrm{~mm}$ de largo, aplanados y alados distalmente; lámina elíptica a orbicular, de 4 a 12 por 3 a $9 \mathrm{~mm}$, coriácea, menudamente papilosa, amarillento-verde, redondeada en el ápice, mucronula$\mathrm{da}$, la base cuneada y decurrente, el margen entero. Inflorescencia terminal, simple, cada flor subtendida por 4 brácteas lanceoladas, 1 a $3 \mathrm{~mm}$ de largo. Flores 4-meras, (raramente 5-meras), pedicelos 2 a $4 \mathrm{~mm}$ de largo. Hipanto 3 a $5 \mathrm{~mm}$ de largo, cáliz 4( a 5)-lobado, lóbulos foliáceos, estrechamente triangulares, carinados, de 3 a $6 \mathrm{~mm}$ de largo, erectos, con glándulas marginales rojas. Corola rosada, embudada, 2 a $3.8 \mathrm{~cm}$ de largo, 1.5 a $2.8 \mathrm{~cm}$ de diámetro distalmente, glabra, tubo 1.6 a $3 \mathrm{~cm}$ de largo, lóbulos triangular-ovados, 8 a 12 $\mathrm{mm}$ de largo. Estambres 4 (o raramente 5), inclusos, 2 más largos con anteras de $6 \mathrm{~mm}$ de largo y filamentos de $1 \mathrm{~cm}$ largo y $0.2 \mathrm{~mm}$ de ancho, en los 2 ( a 3) estambres más cortos las anteras $5 \mathrm{~mm}$ de largo, filamentos de $4 \mathrm{~mm}$ de largo y $0.4 \mathrm{~mm}$ de ancho, anteras lineares, basifijas. Estilo filiforme, glabro, 1 a $2.5 \mathrm{~cm}$ de largo, estigma 2-lobado. Cápsula oblonga, de 8 a 10 por 4 a $5 \mathrm{~mm}$, 2-locular, comprimida, parda y glabrada con dehiscencia septicida, lóbulos del cáliz persistentes; placenta central. Semillas superiores e inferiores 2 a 5 en cada lóculo. Semillas verticales, elípticas, de 3 a $4 \mathrm{~mm}$ por 2 a $2.5 \mathrm{~mm}$, pardas, engrosadas en el margen, la testa reticulada.

\section{Lorencea Borhidi 2003}

Lorencea Borhidi, Acta Bot. Hung. 45: 17 (2003). Tipo: Lorencea guatemalensis (Standl.) Borhidi)

Árbol mexicano y guatemalteco de altura mediana, 9 a 19 m alto; ramitas glabras resinosas. Estípulas intrapetiolares rígidas, connadas en una vaina corta en la base, anchamente deltadas, acuminadas y cuspidadas en el ápice, glabras por fuera, por dentro con coléteres resinosos y digitados. Hojas opuestas, lámina obovado-elíptica, cartácea, discolor, nervios laterales barbados en las axilas del envés. Inflorescencias axilares o raramente terminales, paucifloras, cimoso-paniculadas 2 a 3 veces ramificadas, foliosas, pedunculadas, pedicelos bracteolados con brácteas estpiluformes, lobadas y resinosas. Flores grandes, usualmente 5-meras, a veces 4-meras. Hipanto ánguloso, lóbulos del cáliz 


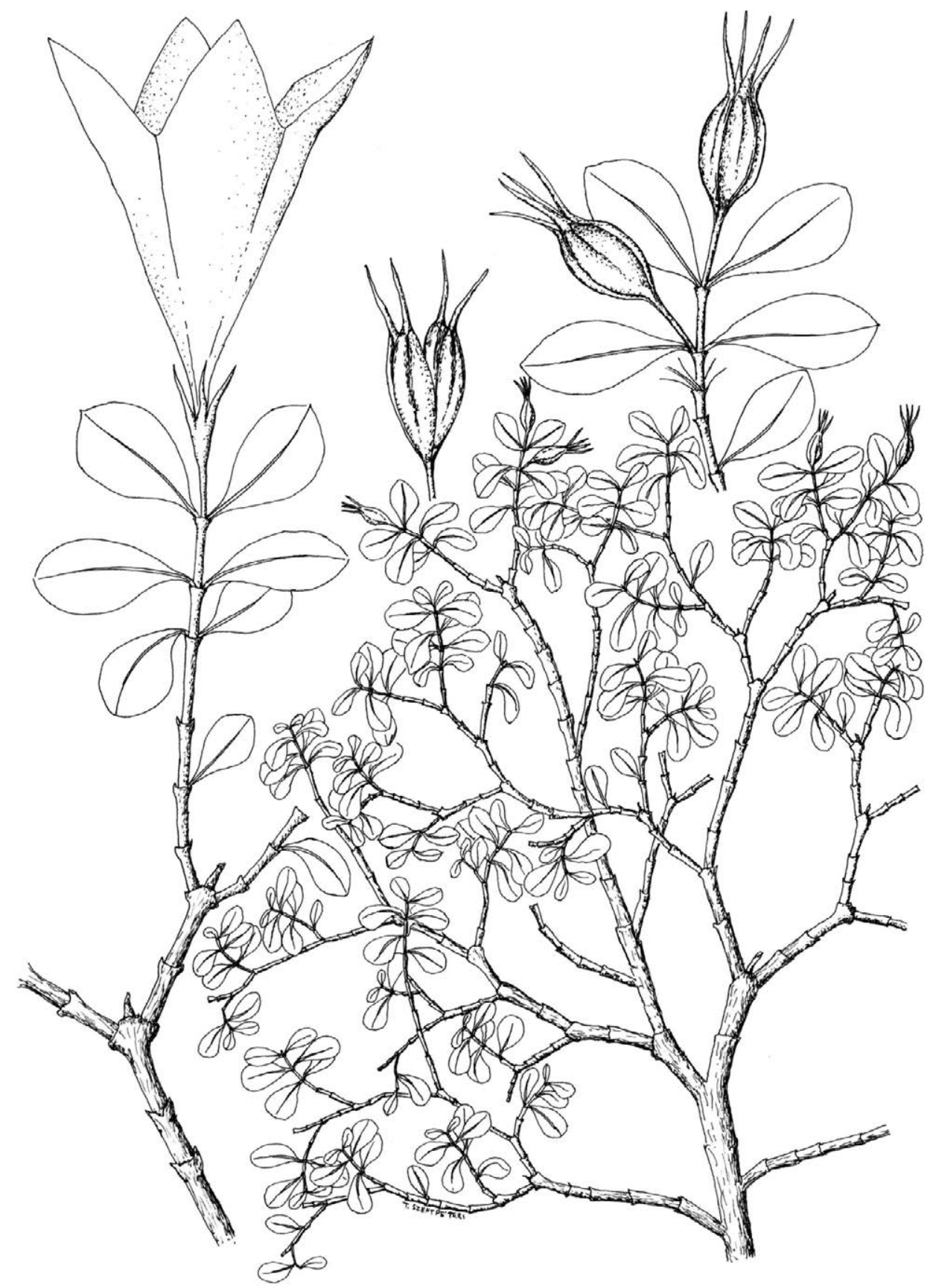

Fig. 6. Coutaportla pailensis Villareal (aspecto general, rama con flor, fruto abierto, dibujo de T. Szentpéteri) 
iguales, subulados, carinados por fuera, canaliculados e hirsutos por dentro. Corola blanca, infundibuliforme, glabra en ambas caras, lóbulos induplicadoimbricados en la yema, más cortos que el tubo. Estambres inclusos, anteras basifijas, flechadas en la base; filamentos hirsutos en la base, connados entre sí y adnados a la base del tubo; estilo filiforme, glabro; estigma corto, 2-lobado. Fruto en cápsula 2-locular, subgloboso, comprimido y costado, lóbulos del cáliz persistentes, dehiscencia septicida; placenta espongiosa, linear, estipitada, erecta, saliendo de la base del lóculo, óvulos verticales. Semillas pocas por lóculo; la testa reticulada, células densamente tuberculadas.

1. Lorencea guatemalensis (Standl.) Borhidi, Acta Bot. Hung. 45: 17 (2003) - (Fig. 7) - Bas.: Portlandia guatemalensis Standl., J. Wash. Acad. Sci. 18: 162 (1928). $\equiv$ Coutaportla guatemalensis (Standl.) Lorence, Syst. Bot. 11: 210 (1986). - Tipo: Guatemala, Alta Verapaz, Quebradas Secas, 2500 ft., 1 Jun 1920, H. Johnson 282; holotipo: US 1081354 n.v., foto: MEXU! - Distr.: México (Chiapas, Veracruz?) y Guatemala.

Árbol mediano, 9 a $19 \mathrm{~m}$ de alto, a veces arbustivos, ramas cilíndricas, glabras, verdoso-pardas. Estípulas intrapeciolares, rígidas, persistentes, anchamente deltadas, de 3 a $6 \mathrm{~mm}$ por 4 a $6(10) \mathrm{mm}$, acuminadas o cuspidadas en el ápice de 1.5 a $3 \mathrm{~mm}$ de largo, glabras por fuera y resinosas con coléteres digitados de 0.3 a $0.5 \mathrm{~mm}$ de largo por dentro. Hojas opuestas, pecioladas, con pecíolos iguales por pares, glabras, de 1 a $3 \mathrm{~cm}$ de largo, alados hacia el ápice; lámina obovado-elíptica o elíptica de 11 a 22 por 3 a $9 \mathrm{~cm}$, aguda y decurrente en la base, acuminada en el ápice con un acumen de 7 a 12 mm de largo, cartácea o subcoriácea, discolor, glabra y nitídula en el haz, domaciada y barbada en las axilas de los nervios laterales en el envés. Estos 7 a 10 pares, ligeramente impresos en el haz, prominentes y amarillentos en el envés con venación visible hasta los nervios terciarios en ambas caras. Inflorescencias axilares o rara vez terminales cimoso-paniculadas, hojosas y 2 a 3 veces ramificadas, de 13 a 29 por 4 a $6 \mathrm{~cm}$, glabras; pedúnculo de 8 a $15 \mathrm{~cm}$ de largo, ejes primarios de $1.5 \mathrm{a} 12 \mathrm{~cm}$ de largo, subtendidos por bractéolas estipuliformes, lobuladas y resinosas. Flores mayormente 5-meras, a veces 4-meras en pedicelos de 3 a 5 mm; hipanto obcónico, 5 ó 4-anguloso, 1.5 a 2 mm de largo, el limbo del cáliz 1 $\mathrm{mm}$ de alto, lóbulos iguales, subulados, rígidos, erectos, de 6 a $10 \mathrm{~mm}$ por 1.2 a $1.8 \mathrm{~mm}$, carinados y glabros por fuera, canaliculados e hirsutos por dentro con margen convoluto. Corola blanca, induplicado-imbricada en la yema, infundibuliforme en la antesis de 2.5 a $3.5 \mathrm{~cm}$ de largo y de mismo ancho arriba, glabra en ambas caras, lóbulos ovados o deltados ca. mitad de largos que el tubo. Estambres 5 o rara vez 4, inclusos, llegando hasta la base de los lóbulos, anteras de $1 \mathrm{~cm}$, linear-subuladas, basifijas, flechadas en la base; filamentos 8 a $10 \mathrm{~mm}$ de largo, hirsutos en la base y connados entre sí hasta $0.5 \mathrm{~mm}$ de largo, y adnados a la base del tubo; estilo filiforme, glabro, de 1.7 a $2.2 \mathrm{~cm}$ de 


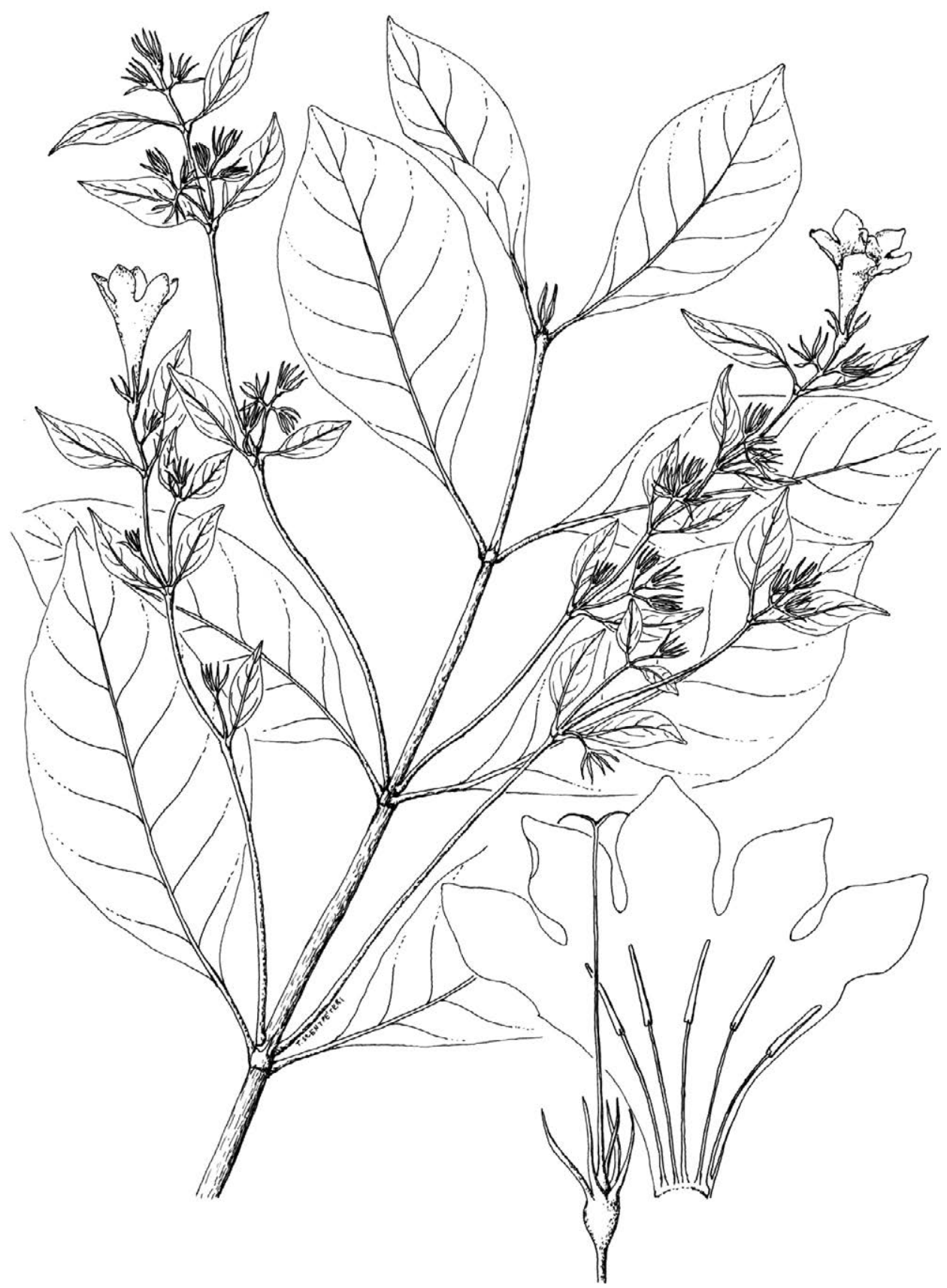

Fig. 7. Lorencea guatemalensis (Standl.) Borhidi (rama con inflorescencias, corola abierta, hipantio con pistilo, dibujo de J. L. Szentpéteri) 
largo, estigma brevemente 2-lobado. Fruto en cápsula 2-locular subgloboso, lateralmente comprimido, de 1 a 1.4 por 1.2 a $1.6 \mathrm{~cm}$, ligeramente 6-costado, con lóbulos del cáliz persistentes, con una dehiscencia ligera loculicida temprana, seguida con una apertura profunda septicida; placenta espongiosa, linear, estipitada, erecta, saliendo de la base del lóculo, óvulos verticales. Semillas pardas, 4 a 5 por lóculo, discoideas o anchamente elipsoidales, de 6 a 8 por 4.5 a $6.5 \mathrm{~mm}$, con un margen pálido y engrosado; la testa reticulada, células densamente tuberculadas.

\section{BIBLIOGRAFÍA}

Aiello, A. (1979): A re-examination of Portlandia (Rubiaceae) and associated taxa. - J. Arnold Arb. 60: 38-126.

Borhidi, A. (1982): Studies in Rondeletieae (Rubiaceae) III. The genera Rogiera and Arachnothryx. - Acta Bot. Acad. Sci. Hung. 28: 65-72.

Borhidi, A. (2003): Lorencea, gen. nov. (Condamineae, Rubiaceae) de Guatemala y México. - Acta Bot. Hung. 45: 13-21. https://doi.org/10.1556/abot.45.2003.1-2.2

Borhidi, A. (2006): Rubiáceas de México. - Akadémiai Kiadó, Budapest, 512 pp.

Borhidi, A. (2012): Rubiáceas de México. Segunda y ampliada edición. - Akadémiai Kiadó, Budapest, 608 pp.

Borhidi, A. y Fernandez, M. Z. (1981a): Studies in Rondeletieae (Rubiaceae) I. A new genus: Roigella. - Acta Bot. Acad. Sci. Hung. 27: 309-312.

Borhidi, A. y Fernandez, M. Z. (1981b): Studies in Rondeletieae (Rubiaceae) II. A new genus: Suberanthus. - Acta Bot. Acad. Sci. Hung. 27: 313-316.

Borhidi, A. y Járai-Komlódi, M. (1983): Studies in Rondeletieae (Rubiaceae) IV. A new genus: Javorkaea. - Acta Bot. Acad. Sci. Hung. 29: 13-27.

Borhidi, A., Járai-Komlódi, M. y Moncada, M. (1980): Acunaeanthus, a new genus of Rubiaceae. - Acta Bot. Acad. Sci. Hung. 26: 279-289.

Bremer, B. (2009): A review of molecular phylogenetic studies of Rubiaceae. - Ann. Mo. Bot. Gard. 96: 4-26 https://doi.org/10.3417/2006197

Delprete, P. G. (1999): Rondeletieae (Rubiaceae), part 1. - Flora Neotropica Monograph 77: $1-226$.

Govaerts, R. (2016): World checklist of Rubiaceae. - Royal Bot. Gard. Kew. http://apps.kew. org/wcsp

Govaerts, R., Frodin, G. D., Ruhsam, M., Bridson, D. M. y Davis, A. P. (2006): World checklist and bibliography of Rubiaceae. - Royal Bot. Gard. Kew, England. http//www.rbgkew. org.uk/data/rubiaceae/index/htm

Lorence, D. H. (1986): The identity of Portlandia guatemalensis (Rubiaceae). - Syst. Bot. 11(1): 209-213. https://doi.org/10.2307/2418958

Lorence, D. H. (1999): A nomenclator of Mexican and Central American Rubiaceae. - Mo. Bot. Gard. Press 73: 1-177.

Lorence, D. H. y Taylor, C. M. (2012): Rubiaceae. - In: Davidse, G., Sousa, M. S., Knapp, S. y Chiang, F. (eds): Flora Mesoamericana. Vol. 4, part 2, pp. 1-288.

Manns, U. y Bremer, B. (2010): Towards a better understanding of intertribal relationships and stable tribal delimitations within Cinchonoideae s.s. (Rubiaceae). - Mol. Phyl. Evol. 56: 21-39. https://doi.org/10.1016/j.ympev.2010.04.002 
Martinez, E., Ramos, C. H. A. y Chiang, F. (1994): Lista floristica de la Lacandona, Chiapas. - Bol. Soc. Bot. Mex. 54: 99-177.

Motley, T. J, Wurdack, K. J. y Delprete, P. G. (2005): Molecular systematics of the CatesbeeaeChiococceae complex (Rubiaceae) flower and fruit evolution and biogeographic implications. - Amer. J. Bot. 92: 316-329. https://doi.org/10.3732/ajb.92.2.316

Ochoterena, H. (2012): 31. Coutaportla Urb. syn.: Lorencea Borhidi. - In: Davidse, G., Sousa, M., Knapp, S. y Chiang, F. (eds): Flora Mesoamericana. Vol. 4, part 2, p. 69.

Rova, J. H. E. (1999): Rubiaceae phylogeny based on rps16 sequence data. - Diss. Bot. Inst. Göteborg Univ. (Suppl. II): 1-29.

Rova, J. H. E., Delprete, P. G., Andersson, L. y Albert, V. A. (1999): Rubiaceae phylogeny based on trnL-F sequence data. - Diss. Bot. Inst. Göteborg Univ. (Suppl. I): 1-35.

Rova, J. H. E., Delprete, P. G., Andersson, L. y Albert, V. A. (2002): A trnL-F cpDNA sequence study of the Condamineae-Rondeletieae-Sipaneeae complex with implications on the phylogeny of Rubiaceae. - Amer. J. Bot. 89(1): 145-159. https://doi. org/10.3732/ajb.89.1.145

Standley, P. C. (1928): New plants from Central America, XI. - J. Wash. Acad. Sci. 18: 160-169.

Standley, P. C. y Williams, L. O. (1975): Rubiaceae. In: Flora de Guatemala, XI(1-3). - Fieldiana Bot. 24(11): 1-274.

Steyermark, J. A. (1967): Rubiaceae. In: Maguire, B. y Wurdack, J. J. (eds): Botany of the Guayana Highland, part VII. - Mem. N. Y. Bot. Gard. 17: 178-285.

Urban, I. (1923): Plantae cubenses novae vel rariores a clo. Er. L. Ekman lectae I. - Symbolae Antillanae 9: 55-176.

Villarreal, J. A. (1987): Coutaportla pailensis spec. nova. - Sida 12: 223. 